\title{
MICROWAVE RADAR DETECTION OF GAS PIPELINE LEAKS
}

\author{
N. Gopalsami ${ }^{1}$, D. B. Kanareykin ${ }^{2}$, V. Asanov ${ }^{2}$, S. Bakhtiari ${ }^{1}$, and A.C. Raptis ${ }^{1}$ \\ ${ }^{1}$ Energy Technology Division, Argonne National Laboratory, Argonne, IL 60439 \\ ${ }^{2}$ AOZT Finn-Trade, St Petersburg, Russia
}

\begin{abstract}
We are developing a microwave radar sensing and imaging system to detect and locate gas leaks in natural gas pipelines. The underlying detection principle is radar backscattering from the index-of-refraction inhomogeneities introduced by the dispersion of methane in air. An essential first step in the development effort is modeling to estimate the radar cross section. This paper describes the modeling results and the experimental efforts underway to validate the model. For the case of leaks from small holes in a pressurized gas pipeline, we modeled the gas dynamics of the leak jet to determine the plume geometry and the variation of methane concentration in air as a function of distance from the leak source. From the static and dynamic changes in the index of refraction in the turbulent plume, the radar backscatter cross sections were calculated. The results show that the radar cross sections of the leak plumes should be detectable by special-purpose radars.
\end{abstract}

\section{INTRODUCTION}

The problem of radar detection of gas leaks from a point source is akin to meteorological targets in weather prediction - both deal with gas-phase media and the volume scattering from dielectric property changes. The source of radar returns in meteorological radars is the change of reflectivity, refraction, and scattering caused by storms, rain, cloud, atmospheric turbulence, and wind movement. Even in the absence of contrasting dielectric materials such as water and ice droplets in the atmosphere, the radars can detect clear-air turbulence and wind shear due to wave scattering caused by index-of-refraction inhomogeneities [1].

The basis of microwave (MW) radar measurements lies in the equation [2]:

$$
P_{r}=\frac{P_{t} G^{2} \lambda^{2} \sigma}{(4 \pi)^{3} R^{4}}
$$

where $P_{r}$ is the received power, $P_{t}$ the transmitter power, $G$ the gain of the transmit/receive antenna, $\lambda$ the wavelength, $R$ the distance to the plume, and $\sigma$ the radar cross section of the plume. The radar cross section of the gas plume depends on a complex dielectric constant denoted by $\varepsilon=\varepsilon^{\prime}-\mathrm{j} \varepsilon^{\prime \prime}$, where the real part, $\varepsilon^{\prime}$, is the relative permittivity [responsible for reflection and refraction $\left(\mathrm{n}^{2}=\varepsilon^{\prime}\right)$ ], and the imaginary part, $\varepsilon^{\prime \prime}$, is the loss factor (responsible for absorption). It also depends on the scattering from the index-of-refraction fluctuation caused by the flow of leak gas [3]. Other factors that can play a role in the radar return include wavelength, incident angle, and polarization diversity.

This paper presents a model for calculating the radar cross section $\sigma$ of leak plumes from a natural gas pipeline. The purpose of modeling is (a) to determine the feasible radar 
range of gas plume detection and (b) to fine tune the radar parameters for optimal detection. The pipeline is assumed to contain methane at a pressure of $50 \mathrm{~kg} / \mathrm{cm}^{2}$. Three classes of leaks are considered, corresponding to 5.4, 17, and $54 \mathrm{~mm}$ holes in the pipe. The model has two parts: (a) gas dynamic model to determine the plume dispersion, methane concentration, and the corresponding index of refraction of methane-air mixture and (b) scattering model based on index-of-refraction inhomogeneities to estimate the radar cross section.

\section{PLUME MODEL}

The leak jet emanating into the atmosphere from a small hole in a pressurized gas pipeline is turbulent and takes the shape of a cone (Fig. 1). The jet flow is characterized by three flow zones dominated by the inertial, intermediate, and buoyancy forces. The flow in each zone is described by the following equations of motion, energy conservation, and plume spread [4]:

$$
\begin{gathered}
\frac{d}{d x} \int_{0}^{\delta / 2} \rho u^{2} \pi y d y=-g \int_{0}^{\delta / 2}\left(\rho-\rho_{a}\right) \pi y d y \\
\frac{d}{d x} \int_{0}^{\delta / 2} u\left(\rho-\rho_{a}\right) \pi y=0 \\
\delta=c \cdot x
\end{gathered}
$$

where $\rho$ is the density of methane-air mixture, $\rho_{\mathrm{a}}$ the density of air, $u$ the velocity of gas, $g$ the acceleration due to gravity, $\delta$ the diameter of the jet, c a constant equal to 0.44 , and $(\mathrm{x}, \mathrm{y})$ are the coordinate variables along the jet axis and lateral to the jet axis, respectively.

Assuming a gas pressure of $50 \mathrm{~kg} / \mathrm{cm}^{2}$ in the pipeline, the initial velocity of the leak flow is $739 \mathrm{~m} / \mathrm{s}$. We considered three leaks with hole diameters of $5.4 \mathrm{~mm}, 17 \mathrm{~mm}$, and 54 $\mathrm{mm}$. Based on Eq. (4), the plume size (diameter) with distance from the leak is given in Fig. 2(a); note that the plume diameter does not depend on the size of the leak hole. We can determine the flow velocities in each zone by introducing in Eqs. (2) and (3) a lateral flow profile given by Schlichting's formula [4]:

$$
u(y)=\left[1-\left(\frac{2 y}{\delta}\right)^{1.5}\right]^{2} u_{m}
$$

where $u_{m}$ is the velocity along the jet axis, and $u(y)$ is the velocity along the lateral axis. Figure 2(b) gives the flow velocity changes along the jet axis as a function of leak distance. 

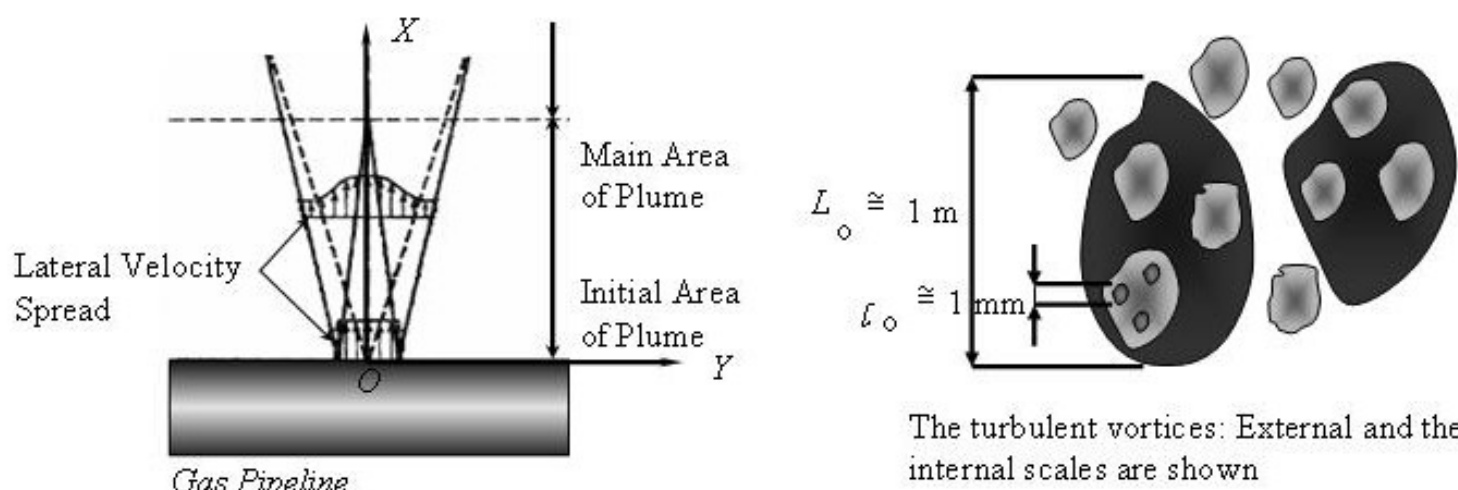

The turbulent vortices: External and the internal scales are shown

FIGURE 1. Plume geometry with turbulent vortices

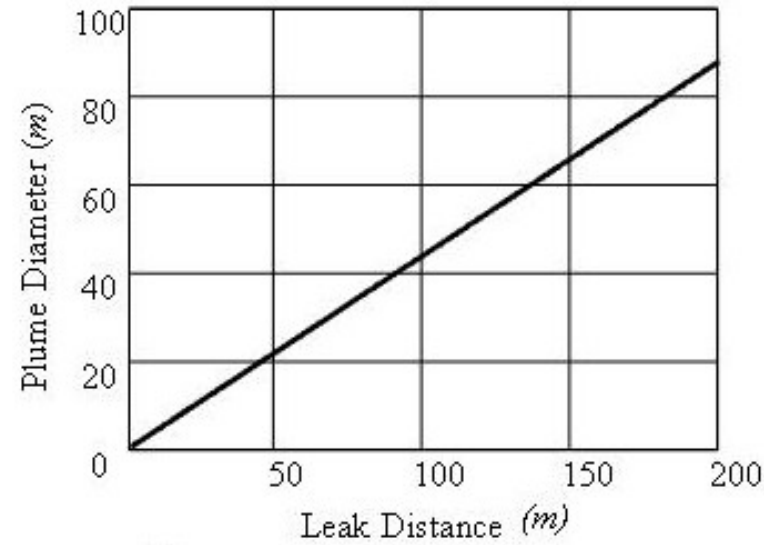

(a)

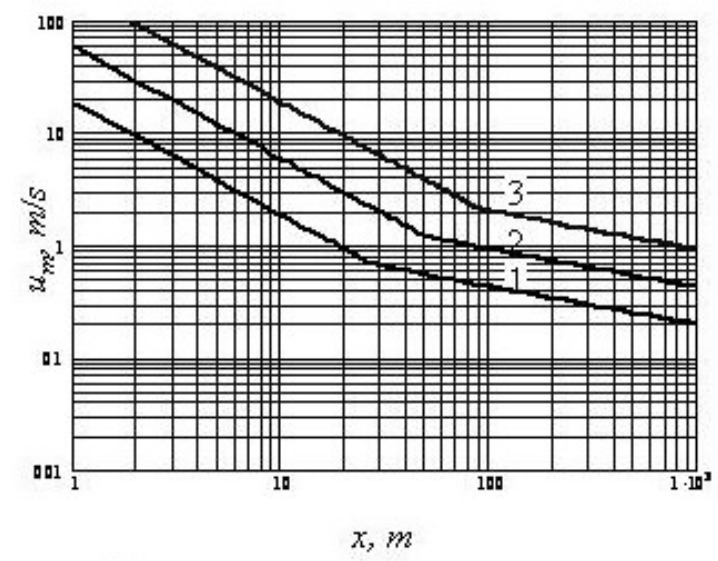

(b)

FIGURE 2. (a) Plume diameter and (b) flow velocity vs. leak distance

Substituting $\rho=\rho_{a}$ at $\mathrm{y}=\delta / 2$ in Eq. (2) and rearranging Eqs. (2)-(4),

$$
\rho_{\mathrm{m}}-\rho_{\mathrm{a}}=\frac{2 \mathrm{~B}}{\mathrm{~A}_{3} \mathrm{u}_{\mathrm{m}} \delta^{2}} .
$$

The normalized density change in the jet is given by

$$
\Delta \bar{\rho}_{m}=\frac{\rho_{m}-\rho_{a}}{\rho_{0}-\rho_{a}}=\frac{u_{0} d_{0}^{2}}{2 A_{3} c^{2} u_{m} x^{2}},
$$

where $\rho_{\mathrm{m}}$ is the density along the jet axis, $\rho_{\mathrm{a}}$ the density of air, $\rho_{0}$ the density of methane, $\mathrm{d}_{0}$ the diameter of the leak hole, and $\mathrm{B}, \mathrm{A}_{3}$, and $\mathrm{c}$ are constants.

The change in the mass concentration due to flow changes in the three zones is shown in Fig. 3 for three holes of diameter $5.4 \mathrm{~mm}, 17 \mathrm{~mm}$, and $54 \mathrm{~mm}$. Thus, from Fig. 2a, the plume diameter is $44 \mathrm{~m}$ at a leak distance of $100 \mathrm{~m}$, and from Fig. 3, the methane concentration is $100 \mathrm{ppm}$ for a $5.4 \mathrm{~mm}$ leak at the same distance. 


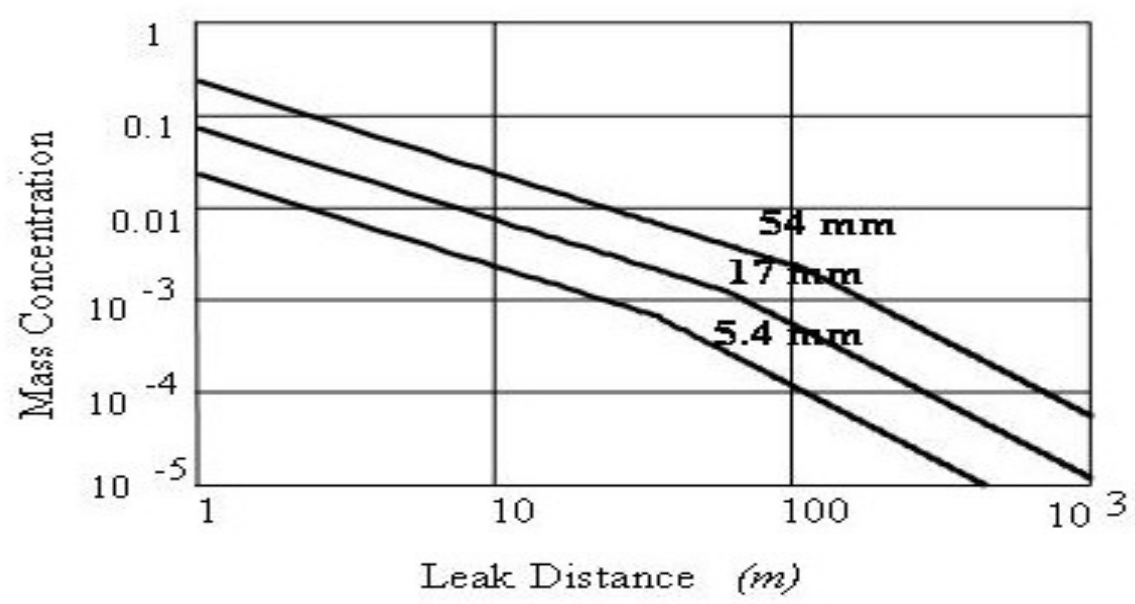

Figure 3. Methane concentration with distance from the leak

\section{RADAR SCATTERING MODEL}

The index of refraction of methane is typically higher than that of dry or moist air. Figure 4 gives the resonance shifts of methane compared to dry air for controlled flow tests performed with a $\mathrm{TE}_{011} \mathrm{MW}$ cavity. The measured values of permittivity for methane and dry air are 1.000671 and 1.000573, respectively; they will be even higher when the cooling effect is considered due to adiabatic expansion of the gas in the jet. From the concentration of methane, we determined the index of refraction of methane-air mixture and plotted it in $\mathrm{N}$ units $\left[=(\mathrm{n}-1) 10^{6}\right]$ in Fig. 5(a). When we take into account the turbulent flow with vortices of a millimeter to a meter scale, the index of refraction fluctuates accordingly. Based on the theory of scattering from the index-of-refraction fluctuations, the specific volume-scattering cross section is given by [5]:

$$
\sigma_{0}=2 \pi \mathrm{k}^{4} \Phi(\mathrm{k})
$$

where $\Phi(\mathrm{k})$ is the spectral density of fluctuations of the index of refraction inside the scattering volume, and $\mathrm{k}$ is the wave number. The spectral density of index of refraction fluctuations according to Kolmogorov's model is given by [6]-[8]:

$$
\Phi(\mathrm{k})=0.033 \mathrm{C}_{\mathrm{n}}^{2} \mathrm{k}^{-11 / 6}, \quad \text { for } 2 \pi / \mathrm{L}_{0} \leq \mathrm{k} \leq 2 \pi / \ell
$$

where $\mathrm{C}_{\mathrm{n}}$ is a structural constant characteristic of fluctuations of the index of refraction of the medium, and $\mathrm{L}_{0}$ and $\ell$ are vortex sizes of external and internal scales, respectively (see Fig. 1). The constant $C_{n}$ is determined from the scale of turbulence and the vertical gradient of the index of refraction.

Figure 5 (b) gives the radar reflectivity (or specific volume-scattering cross section) $\sigma_{0}$ of methane plumes for the three leak classes. The corresponding volume-integrated radar cross sections range from $10^{-9}$ to $10^{-7} \mathrm{~m}^{2}$. These values are generally significantly higher in practice because of possible condensation of water vapor from air due to supercooled gases in the jet.

Equating $\mathrm{P}_{\mathrm{r}}=10$ (receiver noise power) in Eq. (1), we calculated the minimum detectable radar cross section for a $6-\mathrm{kW}, \mathrm{X}$-band marine radar acquired for model verification and leak testing. The results are given in Fig. 6. Because of the fourth-power 
dependence of the radar signal with range, the target must be nearer to the transceiver to detect smaller cross sections. Figure 7 also shows the radar cross sections of methane plumes in comparison with other standard targets. For the chosen radar, the curve for minimum detectable radar cross section shows that the methane plume is detectable at near ranges. The distance of detection can be significantly increased by using high-power transmitters, low-noise receivers, and signal averaging. The estimated scattering cross sections of methane leak plumes, although small, are above the range of normal atmospheric fluctuations of wind, humidity or density, and are detectable by specialpurpose radars tuned to volume-scattering targets.
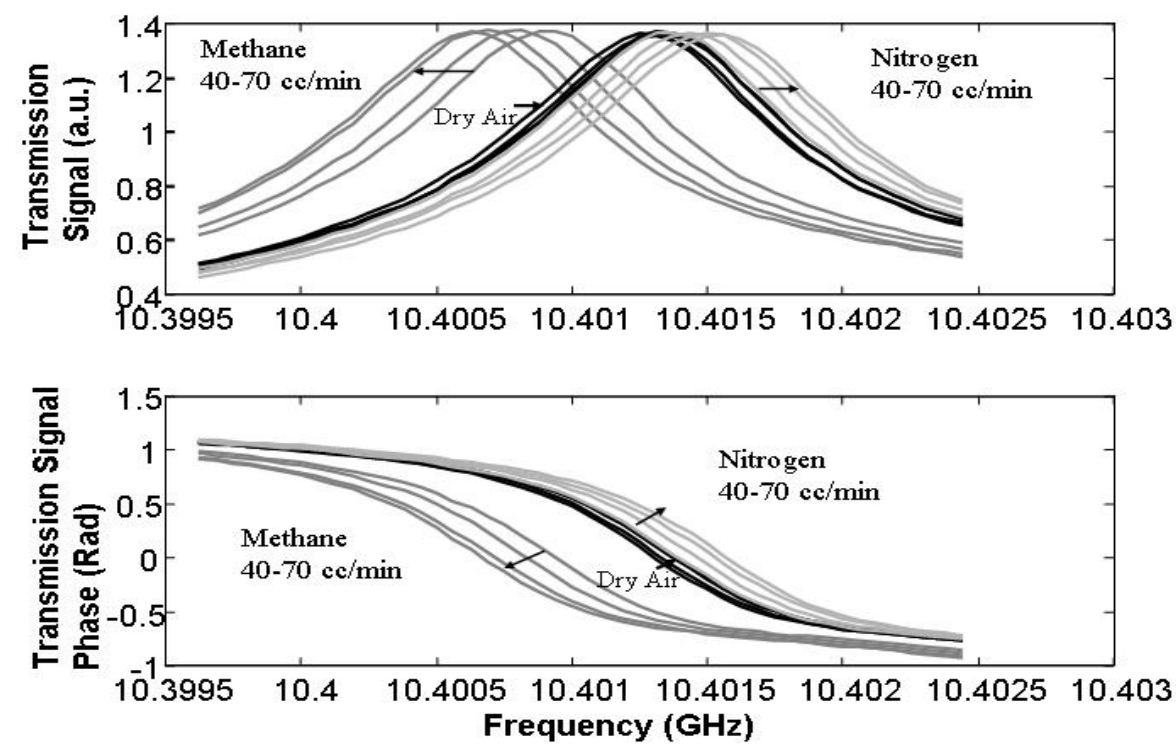

FIGURE 4. Resonance shift of microwave cavity signals for methane, nitrogen, and dry air

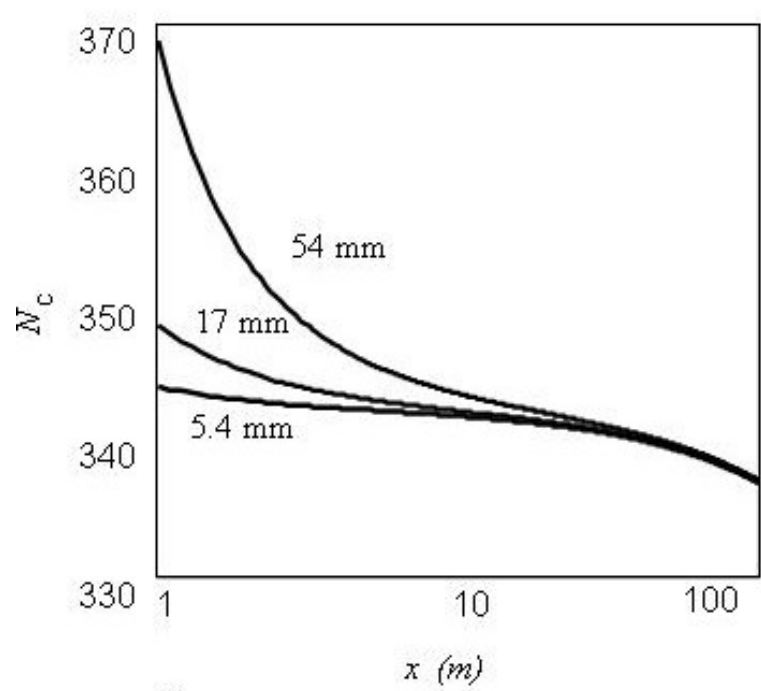

(a)

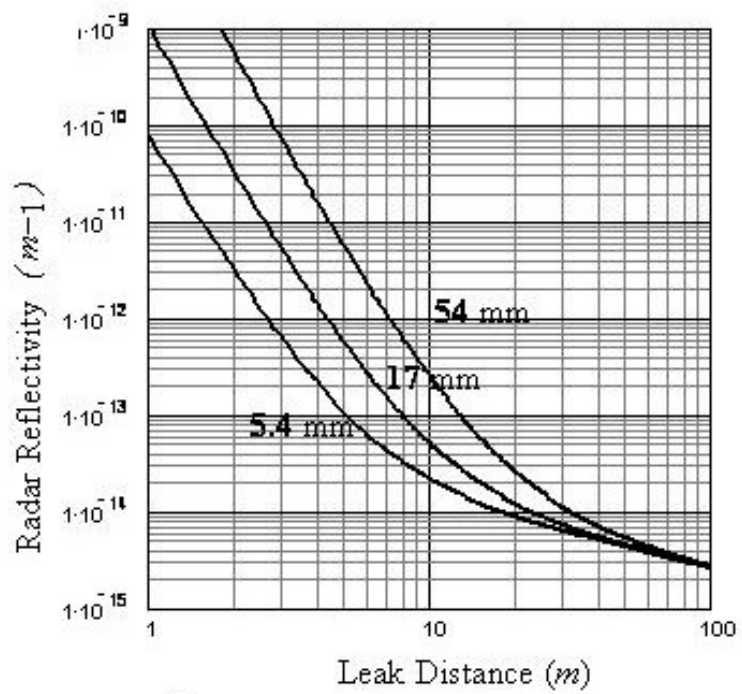

(b)

FIGURE 5. (a) Index of refraction in $\mathrm{N}$ units and (b) radar reflectivity of methane plume vs. leak distance 


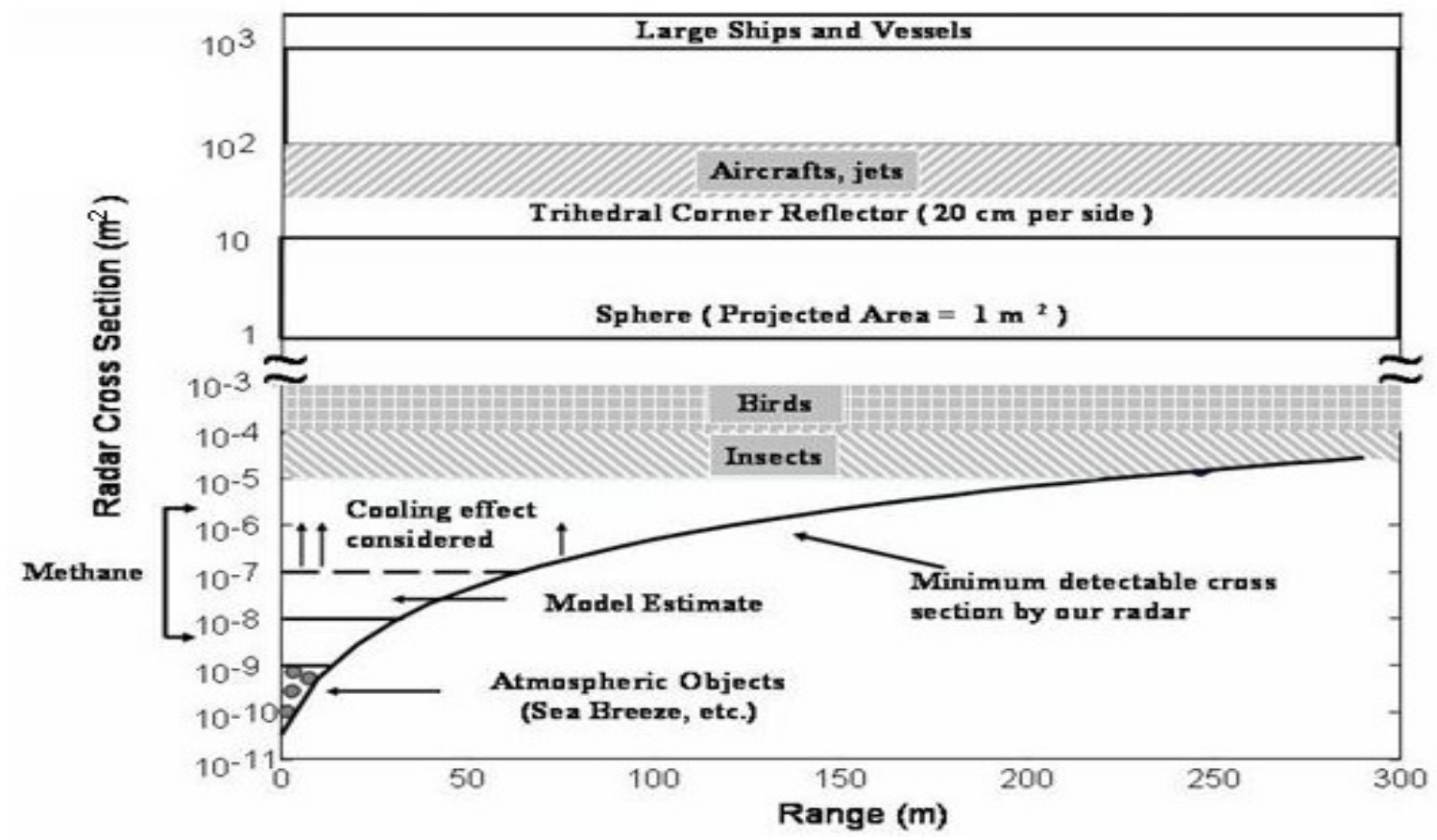

FIGURE 6. Radar cross sections of methane plume and other standard targets

\section{RADAR SETUP FOR LEAK TESTS}

To validate the model and to test leak sensitivity, we installed a 6-kW, X-band pulsed imaging radar on the roof of a shed. The antenna, with a slotted waveguide array, provides a vertical beam width of $22^{\circ}$ and horizontal beam width of $1.9^{\circ}$. We have a $100-\mathrm{m}$ clearance on one side of the radar for leak testing. The radar was calibrated with a corner cube (20 cm per side) situated at $60 \mathrm{~m}$ from the radar. It provides an image resolution of $12 \mathrm{~m}$ along the range and $8 \mathrm{~m} \times 23 \mathrm{~m}$ in the cross range. Figure 7 gives the near-range radar images with and without the corner cube in the scene. Leaks will be set up at the location of the corner cube for radar experiments. Currently, work is in progress to interface the radar unit with a personal computer for data collection and image processing.

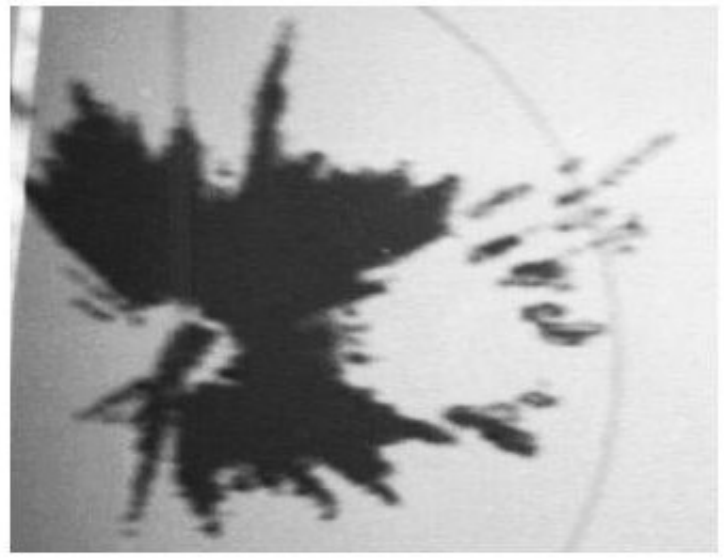

(a)

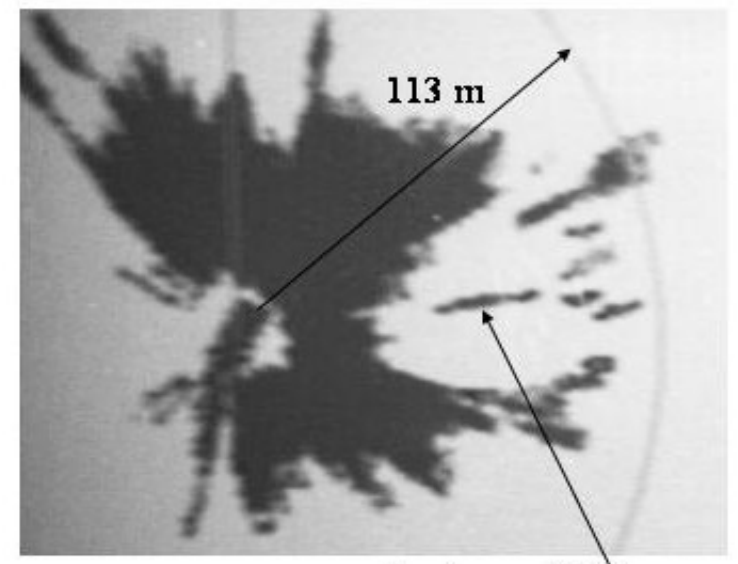

Corner cube $(20 \mathrm{~cm} / \mathrm{side})$ $60 \mathrm{~m}$ away

FIGURE 7. Radar images (a) with and (b) without a calibration target in short range 


\section{CONCLUSION}

Estimation of radar cross section is an essential step in radar testing and imaging of weak targets such as gas plumes. We modeled the leak plumes emanating from small holes in a pressurized gas pipeline. With a gas dynamic model, we determined the geometry of plume dispersion in the atmosphere and the variation of methane concentration in the air surrounding the leak. For a typical gas line, a leak from a hole of a few millimeters forms a turbulent jet in the shape of a cone. The resulting plume spreads to a fairly large size; at a distance of $100 \mathrm{~m}$, for example, the plume diameter is $44 \mathrm{~m}$, and the methane concentration is $100 \mathrm{ppm}$ from a $5.4 \mathrm{~mm}$ leak. A knowledge of the plume geometry is useful in determining the required resolution of the radar.

From the concentration changes of methane-air mixture in the plume geometry, we determined the static and dynamic changes in the index of refraction. With a scattering model based on the inhomogeneities of the index of refraction, we estimated the radar cross section of the plume to be $10^{-9}$ to $10^{-7} \mathrm{~m}^{2}$ for leaks emanating from a $5.4-\mathrm{mm}$ to $54-\mathrm{mm}$ diameter hole. A much higher cross section than predicted by the model is possible, in practice, if one takes into account the cooling effect of an expanding jet and the attendant water vapor condensation. The estimated range of radar cross section lies above the normal fluctuations of the atmosphere and is well within the detectable range of specialpurpose radars. With a combination of a higher-power transmitter, a higher-quality receiver (low noise, high gain), and special signal processing tailored to volume-scattered signals, remote detection of leak plumes from high-flying airplanes is well within the realm of technical feasibility and practicality.

\section{REFERENCES}

1. R. J. Doviak and D. S. Zrnic, Doppler Radar and Weather Observations, New York: Academic Press, 1984.

2. M. Skolnik (ed.), Radar Handbook, New York: McGraw-Hill, 1990.

3. M. Skolnik, D. Hemenway, and J. P. Hansen, "Radar Detection of Gas Seepage Associated with Oil and Gas Deposits," IEEE Trans. on Geoscience and Remote Sensing, vol. 30, pp. 630-633, 1992.

4. G. N. Abramovich, The Applied Gas Dynamics, Moscow: Nauka, 1969.

5. V. Tatarskii, Wave Propagation in Turbulent Medium, New York: McGraw-Hill, 1961.

6. A. Ishimaru, Wave Propagation and Scattering in Random Media, vol. 2, New York: Academic Press, 1978.

7. E. E. Gossard and R. G. Strauch, Radar Observations of Clear Air and Clouds, New York: Elsevier, 1983.

8. P. K. James, "A Review of Radar Observations of the Troposphere in Clear Air Conditions," Radio Science, vol. 15, pp. 151-175, 1980. 